Wiebke Herr und Andreas C. Hofmann

\title{
Podiumsdiskussion „Kollaboration. Interaktion. Die Zukunft geisteswissenschaftlichen Bibliographierens“"
}

DOI 10.1515/bfp-2016-0003

Zusammenfassung: Ende Juni 2015 fand an der Bayerischen Staatsbibliothek (BSB) eine Podiumsdiskussion zu Zukunftsfragen geisteswissenschaftlichen Bibliographierens statt. Anlass war die Präsentation des speziell auf die Didaktik der Geschichte ausgerichteten Bibliographietools ,Index Didacticorum. Research Collaborative Bibliography of History Education“ (I.D.).

Schlüsselwörter: Kollaboratives Bibliographieren; Geisteswissenschaften; Zukunft

\section{Panel Discussion "Collaboration. Interaction. The Future of Bibliographies in the Humanities"}

Abstract: In late June 2015, a panel discussion on questions regarding the future of bibliographies in the humanities took place at the Bavarian State Library (BSB). It was preceded by a presentation of the bibliography tool ,Index Didacticorum. Research Collaborative Bibliography of History Education' (I.D.).

Keywords: Collaborative Bibliographies; Humanities; Future

Am 26. Juni 2015 veranstaltete das Zentrum für Elektronisches Publizieren (ZEP) der Bayerischen Staatsbibliothek (BSB) gemeinsam mit Prof. Dr. Marko Demantowsky (Basel/Brugg-Windisch) und Prof. Dr. Markus Bernhardt (Duisburg-Essen) eine Podiumsdiskussion zu Zukunftsfragen geisteswissenschaftlichen Bibliographierens. Anlass war die Präsentation des speziell auf die Didaktik der Geschichte ausgerichteten Bibliographietools „Index Didacticorum. Research Collaborative Bibliography of History Education“ (I. D.), das an der BSB entwickelt wird und im September 2015 als Betaversion online ging ${ }^{1}$. Unter der

1 http://www.historicum.net/id.

*Kontaktperson: Dr. Wiebke Herr, Wiebke.Herr@bsb-muenchen.de Andreas C. Hofmann, M.A.: Andreas.Hofmann@bsb-muenchen.de
Moderation von Dr. Lilian Landes (BSB) diskutierten insgesamt fünf Experten.

Den Auftakt machte Prof. Dr. Marko Demantowsky (PH FHNW/Universität Basel), der über die Motivation, Konzeption und die Genese des I. D. berichtete. Als Hauptanreiz für eine international ausgerichtete kollaborative Bibliographie zur Geschichtsdidaktik nannte er die Chance, auf diese Weise eine komplette Erfassung auch grauer Literatur und exotischerer Titel zu erreichen. In diesem Ziel läge zudem ein seltenes gemeinsames Interessen seiner ansonsten strukturell kompetitiven Wissenschaftsdisziplin. Dennoch hinge letztlich alles an der ,Gretchenfrage', wie man die wissenschaftlichen Kollegen zum gemeinsamen Bibliographieren motivieren könne. Für das Gelingen einer zentralen kollaborativen Bibliographie der Geschichtsdidaktik hält Demantowsky drei Voraussetzungen für wesentlich: eine technische Niedrigschwelligkeit, ein wissenschaftsaffines Anreizsystem auf der Grundlage von Reputation und eine qualitative Alternativlosigkeit. Vor diesem Hintergrund ging er konkret auf die Rahmenbedingungen und die Umsetzung des I.D. ein: Technische Grundlage ist eine Zotero-Datenbank, welche über eine Schnittstelle in das Geschichtsportal historicum.net eingeblendet wird. Dort können ferner über ein Eingabemodul neue Titel bei der Redaktion zur Freischaltung eingereicht werden. Die klassifikatorische Sacherschließung gewährleistet ein geschichtsdidaktisches Kategoriensystem, welches sowohl ein hierarchisches Browsen durch die Navigation, als auch eine lineare Darstellung mit einer Taglist bietet. Die Gesamtqualität sichert eine mehrköpfige Redaktion. Zur Bekanntheit der Bibliographie tragen bei: die etablierte „Marke“ historicum.net sowie ein gezieltes Offline- und Onlinemarketing. Ein Twitter-Account ist bereits eingerichtet (@IndexDidactico).

Lambert Heller (TIB/UB Hannover) ist Mitglied des an der TIB gegründeten interdisziplinären Teams „Open Science Lab“, das im Kontext des Leibniz-Forschungsverbundes Science 2.0 angesiedelt ist. Er stellte drei Projekte vor: (1) Das an der TIB eingesetzte Forschungsinformationssystem Open Science VIVO beta führt Daten zu Forschungsaktivitäten und -ergebnissen von Hochschulen und anderen Forschungseinrichtungen zusammen. Die Open- 
Source-Software VIVO stellt dabei Forschungsinformationen als Linked Open Data (LOD) bereit. Die Datenhoheit behält der Forscher, die Daten können aber nachgenutzt werden. Dazu zählen auch strukturierte bibliographische Daten. Auf ihrer Basis lassen sich etwa Co-Autorenschaften interaktiv visualisieren und das Publikationsvolumen auf Institutsebene darstellen. Im Sinne eines interdisziplinär orientierten und für zufällige Beobachtungen (Serendipität) offenen „Informationsflaneurs“ (Marian Dörk) werden Beziehungen zwischen Autoren über Titel, Thema, Jahr, Institution etc. abfragbar. Bibliographien würden damit Teil einer weltumspannenden LOD-Cloud. (2) Die TIB beteiligt sich außerdem am CrossRef DOI Event Tracker (DET) Pilot. Dort werden Daten gesammelt, auf deren Basis der Einfluss einzelner wissenschaftlicher Publikationen ausgewertet werden kann. Die Datengrundlage reicht viel weiter als in Almetrics-Konzepten, welche z. B. Nennungen bei Wikipedia, Mendeley oder Twitter mitberücksichtigen. DET Pilot sammelt darüber hinaus Nutzungsdaten, Reviews sowie klassische Zitationen und weist diesen zur eindeutigen und dauerhaften Identifizierung einen Digital Object Identifier (DOI) zu. (3) Ein von der TIB Hannover und der Hochschule Hannover bei der DFG beantragtes Projekt zielt auf die Entwicklung eines Verfahrens zur automatischen Sammlung, Erschließung und Bereitstellung multimedialer Open-Access-Objekte (MOA) mittels der Infrastruktur von Wikimedia Commons und Wikidata. Zusammenfassend stellte Heller die Prognose, dass sich die klassische Bibliographie zukünftig zu einer „Citizen Science“ entwickeln werde, bei der die Rezipienten weit mehr können, als nur zu lesen, zu kopieren und zu importieren. Auf bisherige Literaturverwaltungs-Clients folgten möglicherweise „Executable Bibliographies“, welche das Erheben und Zusammenstellen bibliographischer Informationen deutlich erleichtern könnten.

Matti Stöhr (Jahresberichte für deutsche Geschichte, Berlin-Brandenburgische Akademie der Wissenschaften) gab einen kurzen Abriss funktionaler Standards, Innovationen und Trends im Bereich der Literaturverwaltung. Die klassische Organisation von Literaturnachweisen entwickele sich derzeit $\mathrm{zu}$ einem "Research Management“ weiter, das den gesamten wissenschaftlichen Arbeitsprozess von der Themenfindung, über Informationsbeschaffung und -bearbeitung bis hin zur Textproduktion und Verbreitung begleite und erleichtere. „State of the Art“ seien zudem kombinierte Systeme, welche eine Verbindung von individuellem und kollaborativem Bibliographieren ermöglichen. Stöhr erörterte die Vor- und Nachteile einzelner Softwareanbieter (z. B. Paperpile) und stellte heraus, dass vor allem die Nachnutzbarkeit der Daten etwa in den Bereichen Mindmapping, Visualisierung, Volltextmanage- ment etc. an Bedeutung gewinne, wobei u. a. Standardformate wie BibTeX eine wichtige Rolle spielten. In einem zweiten Teil seines Statements ging er auf die Rolle und Perspektive größerer, institutionell verankerter (Fach-)Bibliographien ein. Eine wesentliche Stärke klassischer Bibliographien sah er in ihrem Anspruch auf Vollständigkeit. Er plädierte dafür, auch zukünftig daran festzuhalten, die Funktion allerdings zu erweitern: Wichtig sei der Einschluss neuer Publikationsformen, wie Blogs, Forschungsdaten etc., die Ergänzung von Bestands- und Zugangsinformationen, die Einbindung in offene Daten- und Informationsinfrastrukturen sowie die Berücksichtigung bibliothekarischer Metadatenstandards. Die Verbindung von automatischen und manuell-intellektuellen Verfahren (auch im Bereich der Sacherschließung) sei dabei wesentlich. Vorteile institutioneller Bibliographien wie Nachhaltigkeit und Zuverlässigkeit würden auf diese Weise mit den Vorteilen „informeller“ Bibliographien wie Flexibilität und Agilität verknüpft. Der DFG-Antrag für einen Fachinformationsdienst Geschichtswissenschaft wählte mit einem Aktionsfeld „Deutsche Historische Fachbibliographie“ einen solchen evolutionären Lösungsansatz.

Dr. Franziska Heimburger (EHESS (École des Hautes Études en Sciences Sociales) Paris) stellte ihre Erfahrungen mit der ,Collaborative Bibliography of the First World War' vor, die sie 2011/2012 mitbegründete. Institutionell ist die Bibliographie bei der International Society for First World War Studies (ISFWWS) angesiedelt. Für den Themenbereich „Erster Weltkrieg“ lag der Ansatz kollaborativen Bibliographierens nahe. Denn im Rahmen der Erinnerungskultur zum hundertsten Jahrestag des Kriegsausbruchs erschien eine sehr hohe Zahl an Publikationen, die der Einzelforscher kaum überblicken kann. Ziel dieser kollaborativen Bibliographie sei jedoch keine Vollständigkeit, vielmehr läge der Fokus auf neuen Veröffentlichungen. Zudem werden neben wenigen Blogs vor allem klassische Publikationsformen verzeichnet. Ausgangspunkt des Projekts war eine Literaturliste von rund 1500 Einträgen, die nach einer von Heimburger erstellten Systematik erschlossen wurde und frei zugänglich gemacht werden sollte. Technische Basis ist eine Zotero-Datenbank, die momentan acht Wissenschaftler direkt bearbeiten können. Andere Beiträger senden Einträge als Google-Formular, das manuell in die Datenbank eingepflegt wird. Nutzbar ist die Bibliographie über die Website der ISFWWS² oder über die entsprechende Zotero-Gruppe ${ }^{3}$. Neueinträge wer-

2 http://www.firstworldwarstudies.org/bibliography.php. 3 http://www.zotero.org/groups/first_world_war_studies_bibliogra phy/items. 
den auch bei Twitter gemeldet. Die Mitarbeit ist ehrenamtlich, das Projekt läuft somit kostenneutral. Nach zunächst zögerlichem Feedback wuchs es signifikant an und über soziale Medien wie Twitter greift inzwischen die Netzwerkfunktion. Exemplarisch führte Heimburger die Möglichkeiten statistischer Auswertungen der Bibliographie vor. So lassen sich etwa die Sprachverteilung der Artikel untersuchen. Zeigt das Ergebnis eine unausgewogene Verteilung, wäre zu hinterfragen, ob sich hierin die tatsächliche Literatursituation widerspiegelt oder die Bibliographie für bestimmte Sprachräume Lücken aufweist. Auch thematisch lasse sich die Bibliographie statistisch auswerten und könne zeigen, welche Themenfelder derzeit am dynamischsten erforscht werden. Für die Zukunft ist u.a. geplant, die Zahl der Mitarbeiter, welche neuen Publikationen direkt in die Bibliographie einpflegen, zu erhöhen und das kollaborative Projekt weiter bekannt zu machen.

In einem Abschlussstatement betonte der zweite Initiator des I.D.-Projektes Prof. Dr. Markus Bernhardt (Universität Duisburg-Essen) die Faszination einer Synthese von neuen Technologien des Internets mit traditionellen Merkmalen einer Bibliographie. Er begrüßte die Genese einer kollaborativen geschichtsdidaktischen Fachbibliographie, die zudem ein schon zu lange bestehendes Desiderat abdecken würde. Für Bernhardt, der sich selbst offenherzig einer „old school“ von Wissenschaftlern zuordnete, sei die „Niedrigschwelligkeit“ des Angebotes von enormer Relevanz. Denn der größte Teil der Wissenschaft könne nur erreicht und zum aktiven Mitgestalten animiert werden, wenn keine technischen Detailkenntnisse vorausgesetzt würden. Als essenziell stufte er die klassifikatorische Sacherschließung ein, die in ihrer Doppelstruktur als hierarchisch-kategoriale und linear-alphabetische Darstellung die „Vollständigkeit“ der Bibliographie doppelt unterstreiche. Auch wenn sich das Tool nicht zuletzt durch die Verwendung englischer Sprache und Fachtermini international positioniert, dürfe es die deutsche Geschichtsdidaktik als ein Kernfeld nicht aus dem Auge verlieren.

Die anschließende Diskussion kreiste um die Frage, inwiefern Maschinen bibliographieren können und wie weit die Schwarmintelligenz reicht. Das Podium diskutierte diese Punkte kontrovers und griff mit unterschiedlichen Aspekten den Komplex der Metadaten auf: Während Heimburger die Qualität redaktionell bearbeiteter und intellektuell ausgewählter Daten betonte, wiesen Heller und Stöhr auf die Vorteile einer automatisierten Vernetzung unterschiedlichster Metadaten hin. Heller hob dabei LODAnsätze und Stöhr die Interoperabilität hervor. Im konkreten Fall fokussierte Demantowsky die Sicherstellung der Homogenität des Angebotes durch eine Redaktion. Bernhardt fügte hinzu, dass die technische Niedrigschwellig- keit ein enormes Maß an Dynamik freisetzen würde. Heller und Stöhr plädierten dafür, durch Nachnutzung bibliographischer Fremddaten, den Aufwand an reiner Tipparbeit zu minimieren. Laut Demantowsky zeige die unterschiedliche Sichtweise unter den Vertretern der informell-kollaborativen Bibliographieprojekte einerseits und denjenigen institutioneller Angebote andererseits, wie positiv es sei, dass am I.D. beide Parteien von Anfang an zusammengearbeitet hätten. Mit Jakob Nielsens „Ungleichheit der Partizipation“ (Beteiligungsrad an Online-Communities: $90 \%$ rezeptiv, 9\% gelegentlich aktiv, 1\% produktiv) wurde die Diskussion dem Publikum übergeben. Dieses diskutierte die Sichtbarkeit und einfache Bedienbarkeit kommerzieller Angebote und die Anforderungen von Geldgebern an ihre Forschungsprojekte. Ein Wettbewerb öffentlicher und kommerzieller Anbieter könne wegen fehlenden Kapitals nicht gewonnen werden. Umso mehr seien freie Verfügbarkeit und Nachnutzbarkeit als ein Wettbewerbsvorteil von Angeboten staatlicher Provenienz zu erkennen. Podium und Publikum diskutierten schließlich wie es gelingen könne, in kollaborativen Bibliographieprojekten zukünftig auf aktive Anreizsysteme verzichten zu können, um einem ,echten` Web 2.0 zu entsprechen. Dabei wurden Aspekte wie die Möglichkeit von PDF-Exporten, Bestellfunktionen und ORCID-Anbindung sowie wissenschaftspolitische Perspektiven auf Reputation und Akzep$\operatorname{tanz}$ thematisiert. Die Veranstaltung wurde gefilmt und ist auf ,L.I.S. A. Das Wissenschaftsportal der Gerda Henkel Stiftung' abrufbar. ${ }^{4}$

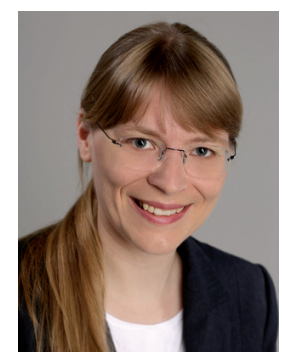

Dr. Wiebke Herr

Bayerische Staatsbibliothek

Ludwigstraße 16

D-80539 München

Wiebke.Herr@bsb-muenchen.de

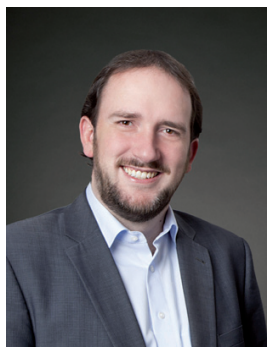

Andreas C. Hofmann, M.A.

Bayerische Staatsbibliothek

Ludwigstr. 16

D-80539 München

Andreas.Hofmann@bsb-muenchen.de

$4 \mathrm{http} / / /$ www.lisa.gerda-henkel-stiftung.de/rcbhe. 